\title{
Imaging and Spectroscopy of Energy-Related Nanomaterials
}

\author{
F. Wang, L.Wu, D.Su, C.Ma, H.Inada, ${ }^{1}$ V.V.Volkov, J.Graetz, J.X. Wang, R.R.Adzic and Y. Zhu \\ Brookhaven National Laboratory, Upton NY 11973 \\ ${ }^{1}$ Hitachi High Technologies America, Inc., Pleasanton, CA 94588
}

The world's demands for energy are expanding at an unprecedented rate. Finding sustainable greenenergy technologies has become one of the most important missions of our nation. The scientific challenge is to discover new ways to efficiently generate, transport, store, and use energies. In the past decade, our group has devoted significant efforts on energy related materials, including superconductors [1], thermoelectrics [2], photovoltaics, fuel cells, and batteries to understand their structure and property relationship. In this presentation, we report our recent work on Li-ion batteries [3] and core-shell nanocatalysts for hydrogen fuel-cell applications [4].

$\mathrm{LiFePO}_{4}$ is one of the most promising cathode materials to power electric vehicles. Direct mapping of the spatial distribution and chemical state of $\mathrm{Li}$ provides critical information on structurecorrelated Li-transport in electrodes. Nevertheless, probing Li, the lightest solid-element on earth, poses an extreme challenge due to its weak scattering power and vulnerability to radiation damage. To meet the challenge we investigated the origins of electron-induced beam damage in Licontaining materials and established the conditions needed to minimize radiation damage and plural scattering. We show, opposite of conventional wisdom, for Li, low voltage operation in TEM (e.g. $60 \mathrm{keV}$ ) causes more damage than high voltage (e.g., $300 \mathrm{keV}$ ) (Fig.1). We explored the possibility of meaningful mapping of $\mathrm{Li}$ and determined its bonding states in the lithiated graphite by comparing the fine structures of typical Li-compounds, such as $\mathrm{Li}, \mathrm{LiF}, \mathrm{LiPF}_{6}, \mathrm{LiOH}, \mathrm{Li}_{2} \mathrm{O}$ and $\mathrm{Li}_{2} \mathrm{CO}_{3}$ to identify different Li-species in the bulk and on the surface. The electronic structure of lithiated graphite retrieved from the near-edge fine structure was then evaluated with ab-initio calculations to shed light on the mechanism of Li intercalation into graphite and the formation of solid-electrolyte-interphases (SEI) [3].

We also studied core $(\mathrm{Pd}) /$ shell $(\mathrm{Pt})$ catalyst particles for oxygen reduction reaction $(\mathrm{ORR})$ in acid fuel cells for electric vehicles [4]. We examined the effects of thickness of the Pt shell, lattice mismatch, and particle size on specific and mass activities for ORR using atomically resolved STEM and EELS. For sphere-like Pd/Pt particles, a dark core and a bright shell are visible when the shell is more than 3-monolayers thick because of the difference of $\mathrm{Pt}$ and $\mathrm{Pd}$ in their atomic number $\mathrm{Z}\left(\mathrm{Z}_{\mathrm{Pt}}=78\right.$, and $\left.\mathrm{Z}_{\mathrm{Pd}}=46\right)$. However, for thinner Pt shells, say a monolayer Pt shell, little contrast is noticeable due to the static and dynamic (Debye Waller factors) displacement of the Pt atoms on the surface that significantly reduces the HAADF intensity. The observations agree well with the multislice calculations based on frozen phonon approximation and EELS measurement (Fig.2). The results demonstrate the importance of microscopy-data interpretation and point a new way to tailoring nanomaterials' properties by controlling their structures [5].

\section{References}

[1] T.M. McQueen, et al., Phys. Rev. Lett. 103, 057002 (2009).

[2] X.. Ke, et al., Phys. Rev. Lett., 103, 145502 (2009).

[3] F. Wang, et al, ACS Nano, DOI: 10.1021/nn1028168 (2011).

[4] J.X. Wang, et al, J. Am. Chem. Soc., 131, 17298 (2009).

[5] Work supported by the U.S. DOE under contract DE-AC02-98CH10886 with funding from BNL-LDRD and DOE/BES-EFRC-NCCES. The fellowship from NSERC of Canada for FW is acknowledged. 

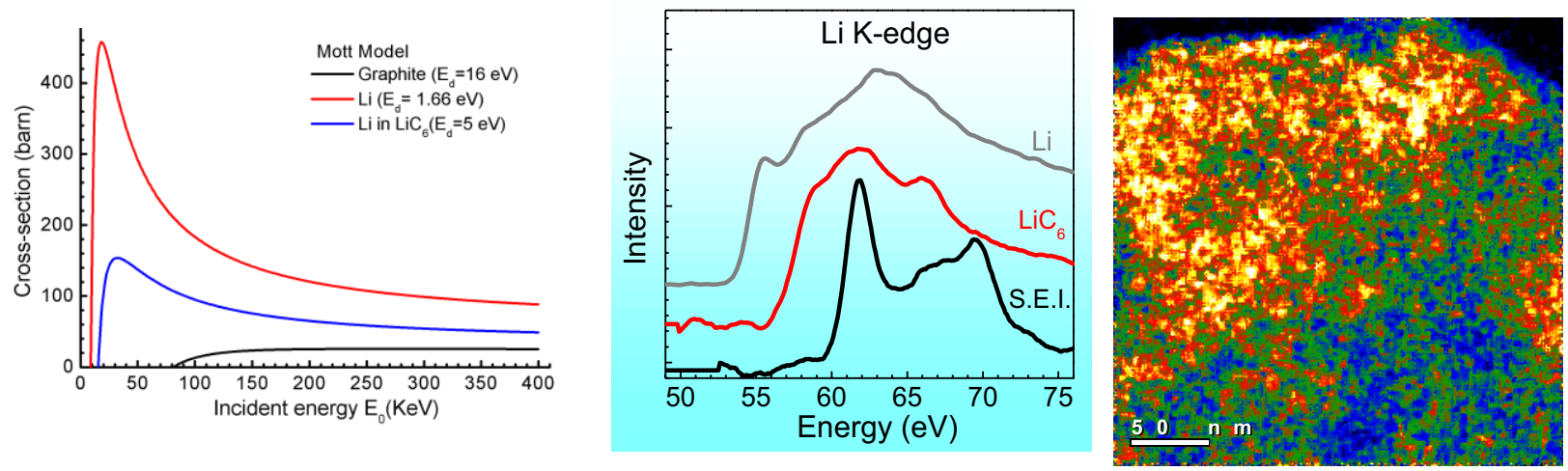

Fig. 1 (Left panel): Calculated displacement cross-sections for elemental Li (red), intercalated Li in graphite, $\mathrm{LiC}_{6}$ (blue), and graphite (black) as a function of incident electron energy $\mathrm{E}_{0}(\mathrm{KeV})$. We demonstrate, opposite of conventional wisdom, for $\mathrm{Li}, 60 \mathrm{keV}$ operation causes more damage than $300 \mathrm{keV}$. (Middle panel): Comparison of $\mathrm{Li}$ bonding states of elemental $\mathrm{Li}, \mathrm{LiC}_{6}$ compound, and the SEI layer. (Right panel): Direct observation of local, non-stoichiometric $\mathrm{Li}$ intercalation in the graphite via $\mathrm{Li}$-mapping using Li-K-edge. Optimized electron energy-loss spectroscopy conditions for Li were used.


Fig.2. (Left panel): (a,b) HAADF-STEM images of the Pd(core)-Pt(shell) nanoparticles obtained for the $\mathrm{PdCPt}_{1}$ and $\mathrm{PdCPt}_{4}$ samples, respectively. We note that the nanoparticles overlap with the others at the left side in (a), and at the top in (b). (c,d) Intensity profiles from the scan lines (the left ends are defined to be zero on the $x$-axes and the intensity at the nearby vacuum set the zero for y-axes) in (a) and (b), and the best fits (black lines), based on the structure models shown in (e) and (f) for Pd particles with 1 and 3-4 Pt surface layers, respectively. The calculations are convoluted with a Gaussian point spread function. Two additional lines in (c) show the calculations made with all of the Pt surface atoms being removed (green line) or replaced by Pd (blue line). (e) Projection of the one-Pt-layer core-shell structure model along the [101] direction (left), and the atomic arrangement (right) of the (1-1-1) plane at the position indicated by the line in the left. (f) Projection of the structure model with 4 Pt layers on the STEM image plane (left) and the arrangements of atoms in the vertical columns along the scan line (right). (Right panel): HAADF and Pd EELS intensity profiles for a $\mathrm{PdCP}_{2}$ sample. (a) Comparison of HAADF (red) and Pd EELS (green) intensity profiles in a line scan. (b-c) Two-dimensional mapping of HAADF intensity (b) and Pd EELS signal (c) obtained with $0.27 \mathrm{~nm} / \mathrm{pixel}$ resolution. 